\title{
Quand les groupes professionnels se mettent en image
}

Sophie Brouquet, Charles Gadéa et Jean-Paul Géhin

\section{(2) OpenEdition}

\section{Journals}

Édition électronique

URL : http://journals.openedition.org/itti/1275

DOI : 10.4000/itti. 1275

\section{Éditeur}

Université de Poitiers

\section{Référence électronique}

Sophie Brouquet, Charles Gadéa et Jean-Paul Géhin, « Quand les groupes professionnels se mettent en image », Images du travail, travail des images [En ligne], 1 | 2016, mis en ligne le 01 février 2016, consulté le 14 avril 2021. URL : http://journals.openedition.org/itti/1275 ; DOI : https://doi.org/ 10.4000/itti. 1275

Ce document a été généré automatiquement le 14 avril 2021

Images du travail, travail des images 


\title{
Quand les groupes professionnels se mettent en image
}

\author{
Sophie Brouquet, Charles Gadéa et Jean-Paul Géhin
}

1 L'histoire des groupes professionnels est jalonnée d'images matérielles, planes ou en relief, fixes ou animées, qui témoignent de la manière dont ils se voient eux-mêmes ou souhaitent être vus. Ces images sont nombreuses, quelquefois très anciennes et présentent des finalités et des formes diversifiées: sculptures, vitraux, peintures, gravures, photographies, films, dessins, bandes dessinées, documents numériques... Le matériau est très abondant, il peut paraître écrasant, il est en fait rarement abordé sous cet angle. Que disent les images des groupes professionnels? Dans quels contextes et avec quelles intentions sont-elles produites? Que révèlent-elles de la place occupée par les métiers dans le monde social dont ils font partie, de leur position dans la division du travail et dans les échelles de revenu, de pouvoir ou de prestige ? Comment montrentelles les activités de travail et les pratiques professionnelles?

2 Le premier numéro de la revue Images du travail, travail des images est consacré aux diverses formes de présentation de soi réalisées ou commanditées soit par des professionnels seuls, soit par des groupements de gens de métier. Dans cette mise en scène, les professionnels se donnent à voir comme les personnages centraux du «drame social du travail », selon la formule de Hughes ${ }^{1}$. Ils sont engagés dans leur rôle, avec leur tenue, leur gestuelle, leurs outils-accessoires et leurs attributs... Ils produisent ainsi une image qui renvoie à des intentions diverses, que ce soit la valorisation des techniques maitrisées, la mise en avant des savoirs détenus ou encore l'affirmation de compétences spécifiques... Pourquoi les groupes professionnels s'intéressent-ils de longue date à la production des images physiques les représentant? Dans quels objectifs? Avec quels effets? Ce dossier thématique vise à apporter des éléments de réponses à ces questions en croisant les points de vue historique et sociologique

3 Sophie Brouquet (médiéviste, université de Toulouse), Charles Gadéa (sociologue, université de Nanterre) et Jean-Paul Géhin (sociologue, université de Poitiers) ont coordonné ce dossier thématique qui croise les approches disciplinaires et cherche à 
articuler analyse des groupes professionnels et approche iconographique. Ce faisant, il s'inscrit dans la démarche initiée par le colloque international Images du travail, travail des images, le festival Filmer le travail et des groupes de travail spécialisés de l'AFS et de l'AISLF'.

En effet, comme le note Charles Gadéa dans le premier article du dossier («L'idiome figuratif des groupes professionnels»), la sociologie des groupes professionnels n'a jusqu'à présent qu'à peine investi la dimension iconographique de son objet, pourtant particulièrement riche en productions très diverses jalonnant toutes les époques du groupement en métiers. Ce silence est lié à une série d'obstacles que rencontrent, chacune de son côté, aussi bien la sociologie des professions que la sociologie visuelle. $\mathrm{Au}$ croisement de ces deux perspectives, l'auteur propose la notion d'idiome figuratif pour rendre compte de la place de l'image dans la construction et l'affirmation des groupes professionnels... Et aussi du travail de rhétorique professionnelle qui l'accompagne. Ce qui frappe, lorsqu'on aborde de ce point de vue, les images mises en avant par les professions, c'est l'ancienneté et l'intensité de cette production comme la diversité des objectifs poursuivis. Ce que montre clairement les six articles de ce dossier.

La question de l'éthique et de la morale est au cœur de ces productions iconographiques et des rhétoriques professionnelles qui les sous-tendent. Étudiant la grande production d'images (dessins aquarellés et lithographies) au sein des compagnons du tour de France au $19^{\text {ème }}$ siècle, Laurent Bastard (« L'absence du travail dans l'iconographie compagnonnique du XIXe siècle »), lui même directeur du musée du compagnonnage de Tours, note que le travail n'est pas présent dans cette iconographie alors qu'il est au cœur du modèle institutionnel. Le paradoxe n'est qu'apparent : le métier, le travail, sont l'ordinaire du compagnon. Ils sont vécus comme le moyen de s'élever socialement mais c'est le Devoir, l'ensemble des règles, rites et symboles, qui permettent cette élévation sociale et morale. Et c'est elle seule qui est privilégiée par l'image. S'appuyant sur un corpus très récent et original, les making of des cinéastes animaliers, Maxence Lamoureux («Les making of: les professionnels de l'image à l'écran ») fait un constat similaire. Les cinéastes animaliers en se filmant au travail puis en montant les images de leurs activités font des choix forts qui prennent sens et construisent une idée de leur métier. Ils focalisent sur les dimensions éthiques $\mathrm{du}$ groupe professionnel en construction (protection de l'environnement, respect de l'animal sauvage, sauvegarde de la biodiversité) et laissent hors champ la plupart des activités quotidiennes du cinéaste animalier.

Bien souvent, la production iconographique vise à rectifier une image considérée comme négative ou détériorée. Comme le note Sylvain Leteux, («L'image des bouchers : la recherche de l'honorabilité, entre fierté communautaire et occultation du sang »), la profession des bouchers véhicule de longue date une image négative liée au rapport au sang, à la mort et à la violence. En réaction à cette image négative, les bouchers ont cherché à donner une image respectable de leur métier, dès le Moyen Âge et la recherche de respectabilité s'affirme dans la période 1850-1950 quand le boucher parisien se représente avec un tablier immaculé et de plus en plus souvent en costume «bourgeois ». Ce costume très codifié devient à partir des années 1930 un marqueur social fort de la profession, porteur de valeurs politiques conservatrices partagées par les patrons bouchers. Cette volonté de construire une image positive d'un groupe professionnel émergent, celui des consultants en management se retrouve dans l'article 
d'Isabel Bony : "Les consultants et leurs images. Mises en récit et mises en scène d'un groupe professionnel ». En s'appuyant sur les photographies mises en ligne sur les sites internet des firmes de conseil ou sur les réseaux sociaux à usage professionnel, l'auteure montre que ces images constituent une ressource significative pour les firmes tant en termes de construction d'une «façade institutionnelle » (comme le dirait Erving Goffman ${ }^{3}$ ) convaincante vis-à-vis des clients, qu'en termes de contrôle social sur les consultants salariés. Il s'agit d'affirmer et de légitimer la qualité de l'expertise détenue par la profession qui apparaissait à la fois faiblement institutionnalisée et reposant sur des savoir-faire plutôt génériques.

7 Ainsi, la production iconographique peut jouer un rôle tout à fait majeur dans la construction d'un groupe professionnel comme le montre Sophie Brouquet qui analyse l'émergence de la profession des artistes («L'image revendiquée : la prise de conscience d'une dignité des métiers d'art à la fin du Moyen Âge »). Une division de la société établie depuis l'Antiquité, oppose au Moyen Âge, les arts libéraux, les activités intellectuelles, aux Arts mécaniques, les métiers manuels. Ces derniers sont vus comme inférieurs, voire méprisables et réservés aux inférieurs, les laboratores. Cette conception cléricale persiste malgré la diversification des arts mécaniques qui, des laboureurs aux orfèvres, couvrent la quasi-totalité des activités, l'essor des villes à partir du $12^{\text {ème }}$ siècle et la création de métiers ou guildes organisés. Progressivement, une hiérarchie s'établit au sein des arts mécaniques. Elle n'est pas toujours fondée sur la richesse; d'autres critères sont revendiqués comme la noblesse du geste, celle de la matière travaillée, l'utilité du métier et aussi sa maîtrise ou son expertise, comme c'est le cas pour les architectes, les sculpteurs, les orfèvres ou encore les peintres. Ainsi, les artistes sont engagés dans un processus de long terme pour acquérir un statut plus respectable, qui les distingue du groupe des travailleurs manuels et des artisans. Pour ce faire, ces premiers producteurs d'images vont massivement mobiliser les images, imposant petit à petit une image plus valorisée de la profession. Les autoportraits de peintres, souvent masqués sous les traits de saint Luc, témoignent de cette évolution.

En dépit de l'apparente diversité des métiers, des médias et des contextes historiques, l'image demeure un support constant de la visibilité, voire de la revendication des métiers. Dans tous les cas, elle est destinée à proposer une représentation savamment construite et développée qui, ensuite, tend à s'imposer au reste de la société. L'image n'est donc pas seulement un document ou un documentaire mais bien une construction qui interroge l'ensemble des sciences humaines et les invite à la coopération. C'est ce que nous avons voulu faire dans ce premier dossier en réunissant des contributions de plusieurs disciplines, dans l'espoir qu'il incitera d'autres chercheurs à prendre en compte ce caractère heuristique des images. Il semble que notre message ait été entendu, puisqu'un deuxième dossier est en préparation, consacré cette fois, non pas à la manière dont les groupes professionnels se mettent en images, mais à la manière dont ils sont représentés par autrui. 


\section{NOTES}

1. Cf. Hughes Everett, Le drame social du travail, Actes de la recherche en sciences sociales Année 1996 Volume 115 Numéro 1 pp. 94-99.

2. Il s'agit du RT1 (Savoirs, travail et professions) de l'Association française de sociologie et du CR32 de l'Association internationale des sociologues de langue française (Savoirs, métiers, identités professionnelles).

3. Cf. Goffman Erving (1974) Les rites d'interaction, Paris, Les éditions de Minuit. 